\title{
Acute Primary Herpetic Gingivostomatitis In A Child: Strategies for Pain Suppression and to Improve Oral Intake
}

\begin{abstract}
Alcione Barbosa Lira de Farias, Maria da Conceição de Barros Correia, Cláudia Wanderley de Barros Correia, Maria Clara Bezerra Fontes, Valter Romão de Souza Júnior, Niedje Siqueira de Lima, Leonardo Cavalcanti Bezerra dos Santos, Marília Cleide Tenório Gomes, Clara Franciely da Mota Sousa, Alane Tamyres dos Santos and Luciana de Barros Correia Fontes*
\end{abstract}

Department of Clinical and Preventive Dentistry, School of Dentistry, Federal University of Pernambuco, Recife, Brazil

*Corresponding author: Luciana de Barros Correia Fontes, Department of Clinical and Preventive Dentistry, School of Dentistry, Federal University of Pernambuco, Recife, Brazil

\begin{abstract}
This case report describes the management strategies and the evolution of the acute herpetic gingivostomatitis condition in a 3-year-old female child with a focus on suppressing pain and to improve oral intake with approaches to medicine and dentistry.
\end{abstract}

Keywords: Herpect Stomatitis; Drug Therapy; Child

\section{Introduction}

Herpetic gingivostomatitis is a condition that most often results from initial gingiva (gums) and oral mucosa infection with herpes simplex virus type 1 (HSV-1). While herpetic gingivostomatitis is the most common cause of gingivostomatitis in children before the age of 5 , it can also occur in adults. The condition is characterized by a prodrome of fever followed by an eruption of painful, ulcerative lesions of the gingiva and mucosa, and often, yellow, perioral, vesicular lesions. HSV-1 is usually spread from direct contact or via droplets of oral secretions or lesions from an asymptomatic or symptomatic individual. Once a patient is infected with the herpes simplex virus, the infection can recur in the form of herpes labialis with intermittent re-activation occurring throughout life [1]. The pathogenesis of herpetic gingivostomatitis involves replication of the herpes simplex virus, cell lysis, and eventual destruction of mucosal tissue. Exposure to HSV-1 at abraded surfaces allows the virus to enter and rapidly replicate in epidermal and dermal cells. This results in the clinical manifestation of perioral blisters, erosions of the lips and mucosa, and eventual hemorrhagic crusting. Sufficient viral inoculation and replication allow the virus to enter sensory and autonomic ganglia, where it travels intraaxonally to the ganglionic nerve bodies. HSV-1 most commonly infects the trigeminal ganglia, where the virus remains latent until reactivation most commonly in the form of herpes labialis [2]. While most children with primary gingivostomatitis will be asymptomatic, some will experience considerable pain and discomfort and are at risk of dehydration. There are no large, well designed studies to clearly determine appropriate therapy for all children [3]. Professionals who treat children in this age group must be able to diagnose and treat common oral manifestations when necessary and should refer the child to a pediatrician for effective treatment if the presence of any systemic alteration is suspected [4]. Herpetic infections commonly affect the dental profession's anatomical area of responsibility and the diagnosis and management of such infections fall in the purview of oral healthcare providers. To administer competent care to patients with herpetic infections, clinicians must understand the disease, its treatment, the impact the disease or its treatment may have on the patient and the extent to which the presence of a herpetic infection may impact on caregivers in the clinical process [5]. The purpose of this case report was to describe the treatment recommended for a child diagnosed with acute herpetic gingivostomatitis associated with tonsillitis and the ways to suppress pain and to improve oral intake from the perspective of medicine and dentistry. 


\section{Case Report}

Parents of a 3-year-old and female child sought pediatrician due to inflammation in the throat of their daughter, with fever and irritability for two days, then their child feels pain in the mouth, and the drooling starts with the appearance of diffuse lesions in the oral mucosa, complaining of pain and having difficulty feeding. There was the prescription of antibiotics (amoxicillin and clavulanate potassium for oral suspension), anti-inflammatory and antipyretic. Intraoral cleaning with gauze and saline was recommended and the request for a new consultation, to eliminate the possibility of fungal contamination. The diagnosis of acute and viral primary herpetic gingivostomatitis was established (Figure 1). On intraoral examination, gingiva appeared fiery red in color and multiple vesicles were present on the attached mucosa. Multiple vesicles and ulcers were seen along the lateral border and anterior surface of the tongue. Both sided buccal mucosa revealed multiple vesicles. Her parents also complained about his bad breath during this period due to poor oral hygiene. Submandibular lymphatic glands of the kid were enlarged [6]. The pediatric dentistry was consulted because the child persisted with much pain, unable to sleep or eat (Figure 2). There was then the option of laser applications, with faster healing of ulcers and greater pain relief. There was substantial improvement in food, oral hygiene and sleep. The patient will perform control examinations, with simultaneous evaluation by pediatrician and pediatric dentistry.

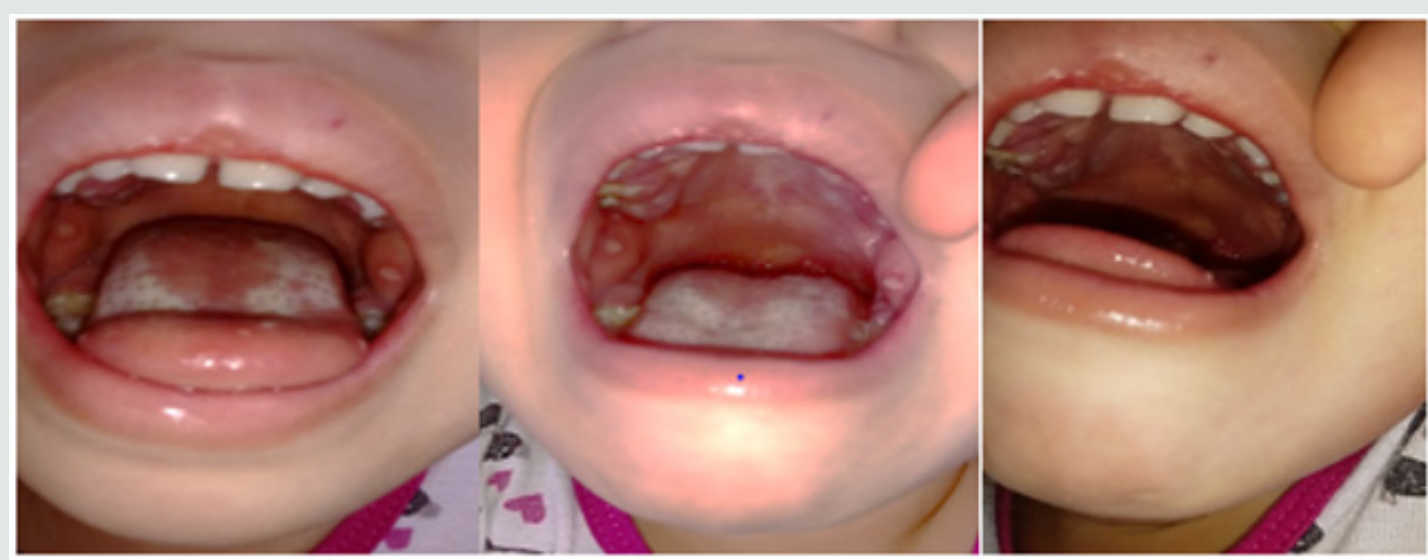

Figure 1: Child oral examination two days under antibiotic prescription.

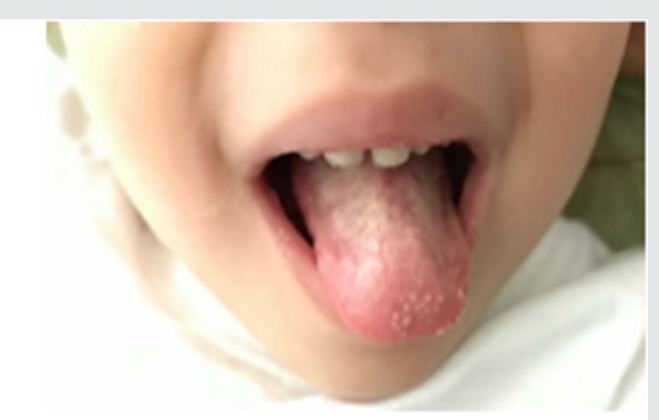

Figure 2: Aspect of the child's tongue on the fourth day of drug treatment.

\section{References}

1. Aslanova M, Zito PM (2019) Herpetic gingivostomatitis. Stat Pearls.

2. Faden $H$ (2006) Management of primary herpetic gingivostomatitis in young children. Pediatr Emerg Care 22(4): 268-269.

3. Goldman RD (2016) Acyclovir for herpetic gingivostomatitis in children. Can Fam Physician 62 (5): 403-404.
4. Padovani MC, Barbosa PS, Baeder F, de Sant Anna GR, Santos MT, et al. (2013) Oral anifestations of systemic alterations in early childhood. J Contemp Dent Pract 14(2): 327-331.

5. Mohan RP, Verma S, Singh U, Agarwal N (2013) Acute primary herpetic gingivostomatitis. BMJ Case Rep 8: bcr2013200074.

6. Karimi M (2019) A review of gingivostomatitis in children. EC Dental Science 18(2): 248-251. 
CC (P) This work is licensed under Creative To Submit Your Article Click Here: Submit Article

DOI: $10.32474 /$ IPDOAJ.2019.03.000153

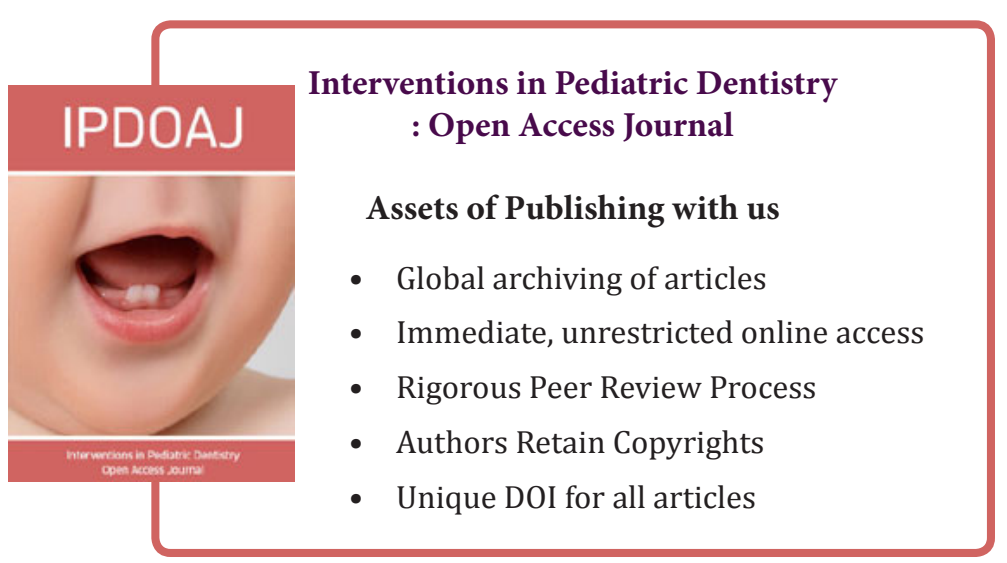

